\title{
No Differences in Early Results of a Hybrid Glenoid Compared With a Pegged Implant
}

\author{
Lawrence V. Gulotta MD, K. Lauchlan Chambers MD, Russell F. Warren MD, \\ David M. Dines MD, Edward V. Craig MD, MPH
}

Received: 2 March 2015/Accepted: 1 September 2015/Published online: 9 September 2015

(C) The Association of Bone and Joint Surgeons (B) 2015

\begin{abstract}
Background Glenoid component loosening after total shoulder arthroplasty is one of the most common causes of failure. A hybrid glenoid that uses peripherally cemented pegs and a central press-fit post may improve implant longevity.

Questions/purposes We asked, compared with polyethylene pegged glenoid implants, do hybrid glenoid implants with a titanium post provide (1) better ingrowth with fewer radiolucencies, (2) better outcome and pain scores, and (3) lower risk of complications and revisions? Methods Between 2009 and 2010, 126 patients underwent primary total shoulder arthroplasty for osteoarthritis.
\end{abstract}

One or more of the authors certifies that he (LVG, RFW, DMD,

EVC), or a member of his or her immediate family, has or may receive payments or benefits, during the study period, an amount of USD 10,000-USD 100,000, from Biomet, Inc (Warsaw, IN, USA).

All ICMJE Conflict of Interest Forms for authors and Clinical Orthopaedics and Related Research ${ }^{\circledR}$ editors and board members are on file with the publication and can be viewed on request.

Clinical Orthopaedics and Related Research ${ }^{\mathbb{R}}$ neither advocates nor endorses the use of any treatment, drug, or device. Readers are encouraged to always seek additional information, including FDAapproval status, of any drug or device prior to clinical use.

Each author certifies that his or her institution approved the human protocol for this investigation, that all investigations were conducted in conformity with ethical principles of research, and that informed consent for participation in the study was obtained.

This work was performed at the Hospital for Special Surgery, New York, NY, USA.

L. V. Gulotta ( $₫)$, R. F. Warren, D. M. Dines, E. V. Craig Hospital for Special Surgery, 535 E 70th Street, New York, NY 10021, USA

e-mail: GulottaL@hss.edu

K. L. Chambers

Coastline Orthopaedics, Fountain Valley, CA, USA
Patients were included in this retrospective study if they consented for inclusion in a shoulder arthroplasty registry, had complete baseline and 2-year data, and had complete radiographs. Eighty-three (67\%) were available at an average followup of 3.2 years (range, 24-45 months). Forty received a conventional all-polyethylene pegged glenoid and 43 received a hybrid component. During the period in question, four of the participating surgeons used only one implant, and four used only the other; there was one highvolume surgeon in each of the study groups. Radiographs were taken at the 2-year followup and analyzed for radiolucent lines. CT scans were obtained randomly for 10 patients with hybrid glenoid implants to assess bone ongrowth. American Shoulder and Elbow Surgeons score, VAS score, complications and revisions were recorded.

Results At final followup, radiolucent lines between the two study groups were not different (hybrid, $1.0 \pm 0.4$; pegged, $1.6 \pm 0.3$; mean difference, 0.6; 95\% CI, 0.85$1.72 ; \mathrm{p}=0.323$ ). Final VAS pain scores were not different (hybrid, $1.2 \pm 0.2$; pegged, $1.5 \pm 0.3 ; \mathrm{p}=0.056$ ). Change in American Shoulder and Elbow Surgeons scores were not different (hybrid, $33.7 \pm 7.3$; pegged, $35.5 \pm 8.2 ; \mathrm{p}=$ $0.283)$. There were no differences in complication risk (hybrid, one of 43 [2.3\%]; pegged, three of 40 [7.5\%]; relative risk, 2.3 ; 95\% CI, 0.82-3.12; $\mathrm{p}=0.061$ ).

Conclusions With the numbers available and at early followup, there were no differences between the hybrid and pegged glenoids in terms of fixation, functional outcome, pain scores, and complications. CT scans confirmed bone ongrowth on the porous titanium post in a small subcohort of patients. Further studies are needed to determine how this new implant will perform with time. Until then, its use should be initiated with caution.

Level of Evidence Level III, therapeutic study. 


\section{Introduction}

Total shoulder replacements have been shown to improve function and diminish pain for patients with end-stage osteoarthritis $[2,10,16,26]$. However, glenoid component loosening remains one of the most common causes of medium and long-term failures [5, 9-12, 15, 18, 22, 23, 25, 27-30, 33]. Attempts to improve glenoid fixation with metal-backed components have been problematic because of accelerated wear, implant loosening, component fracture, screw breakage, and polyethylene dissociation [10, 13, 27, 29]. These findings have led to the conclusion that cemented all-polyethylene pegged and keeled components offer the best chance at prolonged implant longevity and survival. However, one review article suggested that glenoid component loosening still accounts for approximately $39 \%$ of all complications with anatomic total shoulder arthroplasty (TSA) [5]. Methods that improve long-term glenoid fixation will decrease the likelihood of revision and improve functional outcomes.

The ideal glenoid implant would offer excellent initial stability but also would allow for biologic fixation to ensure long-term survival. This is the idea behind a hybrid glenoid design. One such design uses three peripheral polyethylene pegs which are cemented in the bone, combined with a central post made of porous titanium to allow for bone ongrowth (Comprehensive ${ }^{\circledR}$ Total Shoulder System with the Regenerex ${ }^{\circledR}$ Hybrid Glenoid; Biomet, Inc, Warsaw, IN, USA). Ongrowth onto this titanium material has been seen in animal and clinical studies to provide early biologic fixation with bone ongrowth $[1,4,17,21]$. However, to our knowledge, there are no reports regarding its use as a glenoid component.

The purpose of our study was to evaluate the early clinical and radiographic results of a hybrid glenoid implant that uses a central peg consisting of porous titanium designed to promote bone ongrowth, and to compare it with a conventional glenoid implant composed of allpolyethylene cemented pegs. Specifically, we asked: compared with polyethylene pegged glenoid implants, do hybrid glenoid implants with a titanium post provide (1) better fixation with fewer radiolucencies on radiographs, (2) better pain and outcome scores, and (3) lower risk of complications and revisions?

\section{Patients and Methods}

This retrospective study was approved by our institutional review board. The data were prospectively collected through the shoulder arthroplasty registry at the Hospital for Special Surgery. The registry collects demographic and baseline data on the day of surgery for patients undergoing shoulder arthroplasty at our institution. The baseline questionnaire consists of the American Shoulder and Elbow Surgeons score (ASES), VAS score for pain, and basic medical history. The ASES score and VAS score for pain are validated for use in shoulder arthroplasty. Patients then are followed up at 2 years and evaluated for ASES scores, complications, and VAS pain scores. Complications were tallied using the database and cross-checked by reviewing the medical records. The amount of pain was analyzed using the VAS pain score by the patient as measured on a $10-\mathrm{cm}$ strip.

Between January 2009 and June 2010, 126 patients underwent primary TSA for osteoarthritis with an intact rotator cuff and were eligible for inclusion. Twenty-four patients were excluded because they declined to participate in the shoulder arthroplasty registry, and 19 patients had missing baseline or 2-year followup data and were excluded. This left 83 patients who were available for this study (followup rate of $67 \%$ of all TSAs performed). There were 43 patients who underwent TSA using a hybrid glenoid component and 40 who underwent TSA using a conventional all-polyethylene cemented pegged glenoid.

Patients were followed up at an average of 38 months (range, 24-45 months). There were no differences between the hybrid and pegged groups regarding age $(66.3 \pm 8.9$ years in hybrid; $68.2 \pm 9.1$ in pegged; $\mathrm{p}=0.812$ ), sex distribution $(46.5 \%$ female in hybrid; $45.0 \%$ female in pegged; $p=0.551)$, baseline pain $(7.1 \pm 2.1$ VAS in hybrid; $6.9 \pm 1.9$ VAS in pegged; $p=0.714)$, and baseline ASES scores (35.9 \pm 7.1 in hybrid; $32.8 \pm 8.5$ in pegged; $p$ $=0.892)$, proportion involving the dominant arm $(79.1 \%$ in hybrid; $87.5 \%$ in pegged; $p=0.993$ ), and American Society of Anesthesiologists (ASA) class greater than $2(7.0 \%$ in hybrid; $7.5 \%$ in pegged; $p=0.642$ ) (Table 1). Likewise, there were no differences between the patients included in the study, and those who were excluded in terms of age $(67.2 \pm 8.2$ years in the included group; $69.3 \pm 7.1$ years in the excluded group; $\mathrm{p}=0.82)$, sex distribution $(45.8 \%$ female included; $47.9 \%$ female excluded; $\mathrm{p}=0.85$ ), and ASA class greater than 2 (7.2\% included; $9.4 \%$ excluded; $\mathrm{p}=0.64)$. Additional comparisons between the included and excluded cohorts could not be made as data were not available for the excluded patients. Similar numbers of patients were lost to followup in the hybrid group (20 patients) and in the pegged group (23 patients).

The pegged component used in this study was the BioModular ${ }^{\mathrm{B}}$ Total Shoulder System with an all-polyethylene, cemented, pegged glenoid (Biomet, Inc). The hybrid component was the Comprehensive ${ }^{\circledR}$ Total Shoulder System with a Regenerex ${ }^{\circledR}$ Hybrid Glenoid (Biomet, Inc) (Fig. 1). Both implants are approved by the FDA for use in the treatment of shoulder osteoarthritis. Both have a curved back with an anatomic pear shape. The splay of the 
Table 1. Preoperative patient demographics

\begin{tabular}{llll}
\hline Variable & Hybrid & Pegged & p Value \\
\hline Number of patients & 43 & 40 & \\
Age & $66.3 \pm 8.9$ & $68.2 \pm 9.1$ & $0.812^{*}$ \\
Female & $20(46.5 \%)$ & $18(45.0 \%)$ & $0.551^{\dagger}$ \\
Pain VAS & $7.1 \pm 2.1$ & $6.9 \pm 1.9$ & $0.714^{*}$ \\
ASES & $35.9 \pm 7.1$ & $32.8 \pm 8.5$ & $0.892^{*}$ \\
Dominant arm & $34(79.1 \%)$ & $35(87.5 \%)$ & $0.993^{\dagger}$ \\
ASA Class $>2$ & $3(7.0 \%)$ & $3(7.5 \%)$ & $0.642^{\dagger}$ \\
\hline
\end{tabular}

Data presented as either mean $\pm 1 \mathrm{SD}$, or number of patients in the group with the given variable and (percentage); ASES = American Shoulder and Elbow Surgeons score; ASA = American Society of Anesthesiologists score; * Student's t-test; †Fisher's exact test.

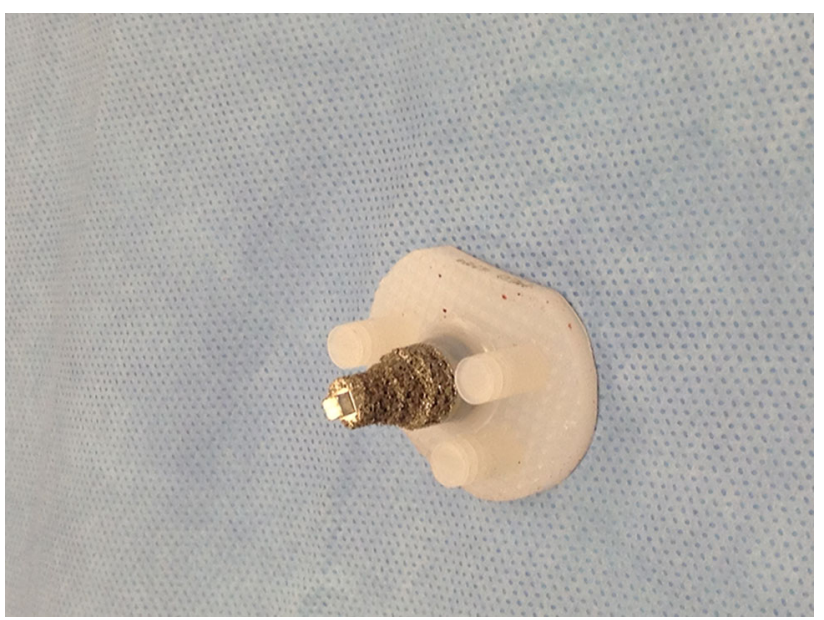

Fig. 1 The Regenerex ${ }^{\circledR}$ Hybrid Glenoid (Biomet, Inc, Warsaw, IN, USA) is shown in this photograph.

peripheral pegs are consistent between the two implants, with the exception of the titanium post in the center of the hybrid component. Both systems allow modularity with any humeral head, therefore allowing varying degrees of radial mismatch. The type of implant was based on the individual surgeon's discretion. Of the eight surgeons participating in the study, four exclusively used the hybrid glenoid (LVG, EVC, RFW, DMD) and the other four exclusively used the pegged glenoid (AA, DA, AP, RM). One surgeon in each group performed more than 50 shoulder arthroplasties a year and was considered "high volume". All procedures were performed in the same institution. They were performed through a deltopectoral approach with a subscapularis tenotomy and repair. Long head of the biceps tenotomy or tenodesis was left to the discretion of the surgeon. Minimal glenoid reaming was used whenever possible, with the exception of shoulders with posterior erosion, in which case the anterior glenoid was eccentrically reamed in an attempt to achieve neutral
Table 2. Glenoid radiolucent line grading systems [10, 20]

\begin{tabular}{|c|c|}
\hline Grade & Description \\
\hline \multicolumn{2}{|c|}{ Pegged component } \\
\hline Grade 0 & No radiolucency \\
\hline Grade 1 & Incomplete radiolucency around 1 peg \\
\hline Grade 2 & $\begin{array}{l}\text { Complete radiolucency }(<2 \mathrm{~mm} \text { side }) \\
\text { around } 1 \text { peg with or without incomplete } \\
\text { radiolucency around one other peg }\end{array}$ \\
\hline Grade 3 & $\begin{array}{l}\text { Complete radiolucency }(<2 \mathrm{~mm} \text { wide }) \\
\text { around } 2 \text { or more pegs }\end{array}$ \\
\hline Grade 4 & $\begin{array}{l}\text { Complete radiolucency ( }>2 \mathrm{~mm} \text { wide) } \\
\text { around } 2 \text { or more pegs }\end{array}$ \\
\hline Grade 5 & Gross loosening \\
\hline \multicolumn{2}{|c|}{ Hybrid component } \\
\hline Grade 0 & No radiolucency \\
\hline Grade 1 & $\begin{array}{l}\text { Incomplete radiolucencies around } \\
1 \text { or more cemented pegs }\end{array}$ \\
\hline Grade 2 & $\begin{array}{l}\text { Incomplete radiolucencies around } \\
\text { the Regenerex }{ }^{\circledR} \text { post }\end{array}$ \\
\hline Grade 3 & $\begin{array}{l}\text { Complete radiolucency }(<2 \mathrm{~mm} \text { wide }) \\
\text { around post }\end{array}$ \\
\hline Grade 4 & $\begin{array}{l}\text { Complete radiolucency ( }>2 \mathrm{~mm} \text { wide) } \\
\text { around post }\end{array}$ \\
\hline Grade 5 & Gross loosening \\
\hline
\end{tabular}

version. Cement technique was similar between groups, with cement pressurized into the peripheral peg holes with digital pressure, and a small amount of cement applied to the back of the implant. Pressure was applied until the cement had hardened.

Standard shoulder AP (Grashey view) and axillary radiographs were evaluated at a minimum of 2 years after surgery. The images were reviewed by two orthopaedic surgeons (LVG and KLC). Radiolucencies were graded according to a system described by Lazarus et al. [20] for the pegged components, and a modification of that system for the hybrid components (Table 2) [10]. Discrepancies between reviewers were settled by consensus.

Ten patients in the hybrid group were randomly selected to have CT scans of their shoulders with 1-mm cuts. CT was used only for 10 patients as a pilot portion of the study to see if bone was growing onto the central titanium post. After a trend was established with the first 10 patients, no additional CT scans were performed so patients would not be exposed to unnecessary radiation. The axial and coronal images were evaluated to assess bone opposition to the central titanium post. An image through the central portion of the titanium post in each of the planes was analyzed. The post then was bisected creating a total of eight surfaces to which bone opposition was judged (Fig. 2). If there was bone opposition in more than six surfaces, the implant was deemed to be solidly fixed. This analysis was graded 

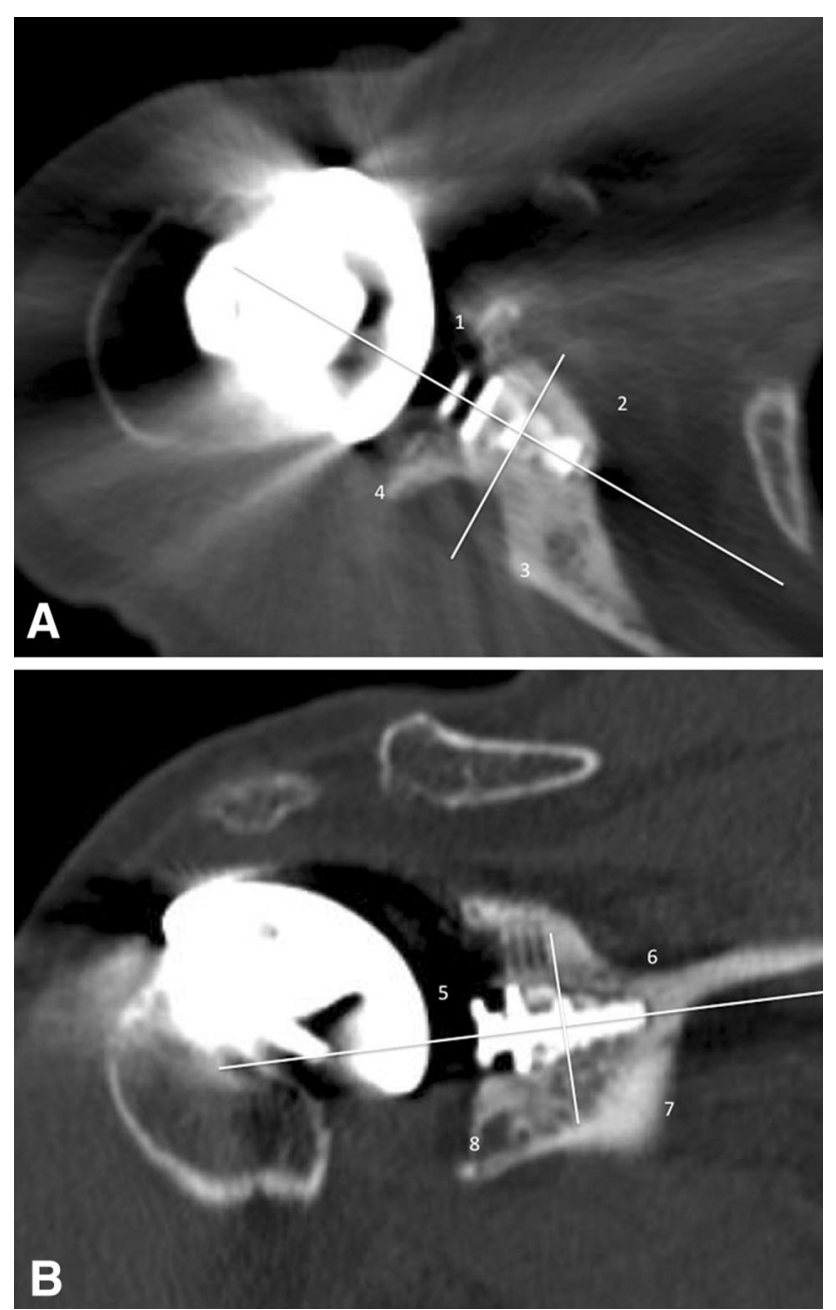

Fig. 2A-B (A) Axial and (B) coronal CT reconstructions of the hybrid glenoid are shown. The central post was divided into a total of eight zones. Bone opposition to the implant in six of the eight zones constituted a solidly fixed implant. In this sample, the only zone in which bone was not appreciated next to the implant was in Zone 6 (Illustration B). Therefore, this implant was deemed to be solidly fixed since seven of the eight zones had bone opposition along the central metal post.

independently by two orthopaedic surgeons (LVG and KLC), and discrepancies were resolved by consensus.

Independent samples and paired t-tests were used to analyze continuous data and Fisher's exact test was used for categorical radiographic data. Significant differences were determined by an alpha value less than 0.05 . Data for the radiolucent line score, ASES score, and VAS pain scores are presented as the mean plus or minus one SD. Before beginning the study, a power analysis was performed for the primary outcome of radiolucent lines observed on the 2-year followup radiographs. Thirty-nine patients in each group were needed to achieve a power of $80 \%$, assuming a minimal clinically significant difference of 1.0 .
Table 3. Outcomes at 2 years

\begin{tabular}{llll}
\hline Variable & Hybrid & Pegged & p Value \\
\hline Radiolucent score & $1.0 \pm 0.4$ & $1.6 \pm 0.3$ & $0.323^{\dagger}$ \\
ASES & $83.5 \pm 13.1$ & $80.1 \pm 10.1$ & $0.331^{\dagger}$ \\
Change in ASES & $38.7 \pm 7.3$ & $35.5 \pm 8.2$ & $0.283^{\dagger}$ \\
Pain VAS & $1.2 \pm 0.2$ & $1.5 \pm 0.3$ & $0.561^{\dagger}$ \\
Change in VAS & $5.8 \pm 0.7$ & $6.0 \pm 0.5$ & $0.911^{\dagger}$ \\
Complication & $1(2.3 \%)$ & $3(7.5 \%)$ & $0.061^{*}$ \\
Reoperation & $1(2.3 \%)$ & $2(5.0 \%)$ & $0.911^{*}$ \\
\hline
\end{tabular}

Data presented as either mean $\pm 1 \mathrm{SD}$, or number of patients in the group with the given variable and (percentage); ASES = American Shoulder and Elbow Surgeons score; * Fisher's exact test; ‘Student's t-test.

\section{Results}

There were no differences in radiolucent line scores between hybrid titanium and all-polyethylene implant designs at final followup. The average radiolucent line score was $1.0 \pm 0.3$ for the hybrid group and $1.6 \pm 0.4$ for the traditional group (mean difference, $0.61 ; 95 \%$ CI, 0.85 $1.72 ; \mathrm{p}=0.323$ ) (Table 3 ). Of the $10 \mathrm{CT}$ scans obtained in the hybrid group, all implants were deemed to be solidly fixed.

Between the hybrid titanium and polyethylene peg groups, there were no differences in changes of outcome scores from baseline and the outcome scores at final followup (Table 3). Final ASES scores were $83.5 \pm 13.1$ in the hybrid group and $80.1 \pm 10.1$ in the pegged group (mean difference, 3.4; 95\% CI, 0.34-1.90; $\mathrm{p}=0.331$ ). Changes in ASES scores from before surgery to final followup were $38.7 \pm 7.3$ in the hybrid group and $35.5 \pm 8.2$ in the pegged group (mean difference, 3.22; 95\% CI, 0.55$1.23 ; \mathrm{p}=0.283$ ). Final VAS pain scores were $1.2 \pm 0.2$ in the hybrid group and $1.5 \pm 0.3$ (mean difference, $0.3 ; 95 \%$ CI, 0.32-1.76; $\mathrm{p}=0.561$ ). Changes in VAS pain scores were $5.8 \pm 0.7$ in the hybrid group and $6.0 \pm 0.5$ in the pegged group (mean difference, $0.2 ; 95 \% \mathrm{CI}, 0.34-1.98$; $\mathrm{p}$ $=0.911$ ).

With the numbers available, there were no differences in the proportion of patients experiencing a complication between groups, as follows: hybrid, one of 43 (2.3\%); pegged, three of $40(7.5 \%)$ (relative risk, $2.3 ; 95 \% \mathrm{CI}$, $0.82-3.12 ; \mathrm{p}=0.061)$. Likewise, with the numbers available, the proportion of patients who underwent a reoperation were: hybrid, one of 43 (2.3\%); pegged, two of $40(5.0 \%$ ) (relative risk, $1.4 ; 95 \% \mathrm{CI}, 0.74-1.62 ; \mathrm{p}=$ 0.911 ). However, our numbers are likely too low to detect a difference should one exist (Table 3). The complication in the hybrid group was posterior instability (one patient); this patient underwent revision of the TSA to a reverse total shoulder replacement approximately 9 months after the 


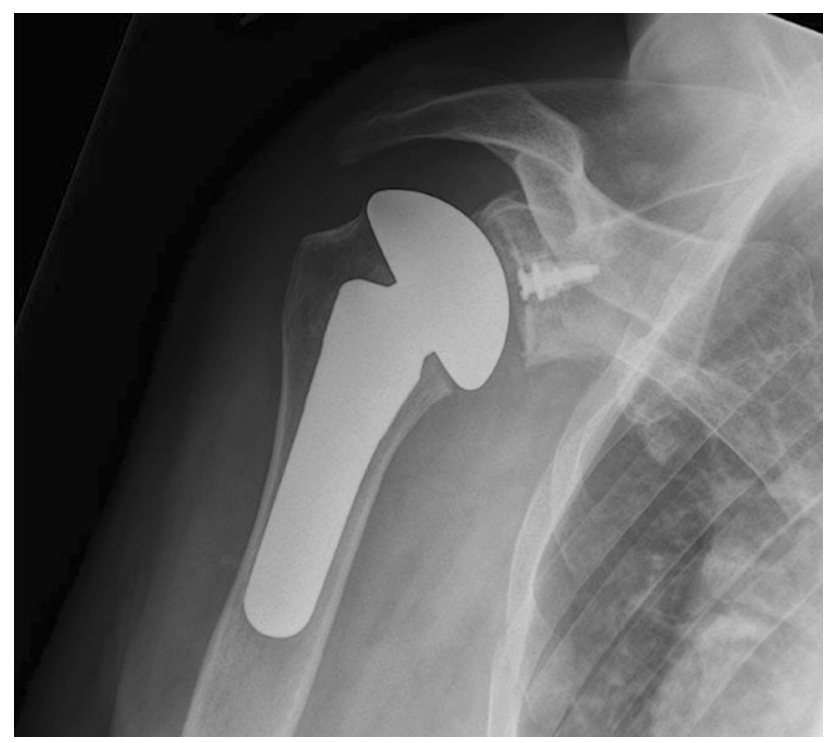

Fig. 3 A postoperative AP radiograph shows a well-functioning total shoulder replacement with the hybrid glenoid.

index procedure. At the time of revision surgery, the central post was found to be solidly fixed in the glenoid bone. It was removed with a trephine and the reverse baseplate was fixed without difficulty (Fig. 3). The complications in the pegged group were two infections in two patients, one superficial and one deep, and persistent biceps tendinitis in one patient. The patient with the superficial infection was treated successfully with antibiotics but the other two patients underwent reoperations, one had irrigation and débridement and one had a biceps tenodesis. None of the complications in either group was deemed to be attributable to the glenoid component used.

\section{Discussion}

The reported incidences of glenoid loosening after TSA varies from $0 \%$ to $12.5 \%$, with some studies reporting rates as much as $96 \%$ if radiolucent lines are considered $[3,5,8$, $10-15,19,20,22,23,30,33]$. This has led to a search for improved glenoid fixation. Early attempts at noncemented, metal-backed glenoid components resulted in unacceptably high failure rates compared with all-polyethylene pegged and keeled components [10, 27, 29]. Because of these findings, cemented polyethylene components now are considered the standard of care. The purpose of our study was to determine how a new hybrid glenoid implant that uses peripherally cemented polyethylene pegs and a central press-fit post performed compared with a conventional allpolyethylene pegged component. Specifically, we aimed to compare the two in terms of (1) fixation as determined by radiolucent lines, (2) functional outcomes as determined by
ASES score and VAS pain score, and (3) complications and reoperations. With the numbers available, and at early followup of an average of 3.2 years, we found that there were no differences between the hybrid and the pegged glenoid components in terms of radiolucent lines, ASES and VAS pain outcomes, and complications and reoperations.

This study has some limitations that need to be considered when interpreting the results. The first is the relatively short followup. Although early glenoid loosening can occur, it is rare. Long-term studies are needed to see if the hybrid implant results in better survival. However, it is important to critically evaluate new technologies at varying times to assess for success and failures. The same holds true for the hybrid glenoid component. Another limitation was our study's design; although the registry is prospectively collected, our analysis is retrospective. This contributed to the amount of patients lost to followup. It is unclear how improved followup would have affected the results because there were no differences in the demographics of the patients included in the study and those who were lost to followup. In addition, similar numbers of patients were lost to followup in the hybrid and the pegged groups. The surgeons grading the radiographs and CT scans were not blinded, as it was impossible to hide the titanium peg when evaluating for radiolucent lines and bone ongrowth, and this could be a source of assessor bias. Only 10 patients with the hybrid component were selected to have CT scans. Once a trend toward boney opposition to the metal post was determined, we thought it was not necessary to subject patients to unnecessary radiation with an otherwise well-functioning implant. It is unclear if different conclusions would be made if the other 33 patients had CT scans. A final potential confounding variable is that several surgeons contributed to the database from which the study was based. This created some variability in how the long head of the biceps was addressed at the time of surgery. However, we believe this makes the results more generalizable to the general orthopaedic community as the surgeons had varying levels of experience with shoulder arthroplasty.

This study showed no differences between the hybrid and the pegged components in terms of fixation as determined by the amount of radiolucent lines around the implant. Gross loosening of the glenoid implant is something that typically occurs with longer followup. Fox et al. [10] reported that glenoid component survivorship can be as much as $99 \%$ at 5 years using all-polyethelene cemented components. Because frank loosening is a relatively rare occurrence, others have evaluated the early development of radiolucent lines to help predict eventual glenoid loosening $[3,6,8,14,19,20,24,30,33]$. Although it might be debatable whether early radiolucencies portend future 
loosening, they generally are regarded as something to be avoided if possible. These findings are consistent with those of similar implants that have been reported in other studies. De Wilde et al. [7] and Vidil et al. [31] have reported their experiences using a noncemented polyethylene glenoid component with three peripheral pegs and a central compression post. Both studies showed that the majority of the implants had bone opposition around the implant on CT scans, and none of the patients had clinical signs of implant loosening. Wirth et al. [32] also reported similar findings. At an average of 4 years after surgery and with 44 patients, they reported that 20 had perfect glenoid seating and no radiolucencies whereas three showed osteolysis around the implant, with the remainder falling between. A major limitation of all of these studies is that they did not include a control group. In our study, the hybrid glenoid implant showed no differences with the numbers available in terms of radiolucent lines when compared with a pegged implant at early followup. A random sample of 10 well-functioning implants showed bone opposition against the metal post in all patients. Whether these findings relate to higher survival rates with time remains to be seen and will be the subject of future studies.

With the numbers available, there were no differences between the hybrid and pegged glenoid groups regarding functional outcomes. The functional outcomes included in the study were ASES score, change in ASES scores from before and after the surgery, VAS pain score, and change in VAS pain scores from before and after the surgery. This is not surprising since the actual bearing surface, geometry, and surgical technique used in the two groups were similar. The only difference between the two groups relates to the backside of the implant, and fixation. Therefore, barring a substantial difference in fixation between groups, it would be difficult to show a functional difference.

There were no differences between the two groups in terms of complications and reoperations. The complication in the hybrid group was for posterior instability and required revision to a reverse shoulder arthroplasty. In the pegged group, one patient had a superficial infection and was treated with antibiotics, one had a deep infection requiring irrigation and débridement, and a third had persistent biceps tendinitis requiring subsequent biceps tenodesis. None of these complications or reoperations was thought to be attributable to the type of glenoid component used.

At early followup and with the numbers available for followup, there were no differences between the hybrid and pegged glenoid groups in terms of fixation, functional outcomes, and complications. As with any new technology, caution must be taken when first using it owing to risks involved in the learning curve, uncertainty regarding how the implant will perform with time, and issues involving costs and value. Therefore, until superiority of the new hybrid implant is shown over traditional implants, they should used where clinical outcomes can be tracked as part of a clinical research program, rather than as part of routine clinical practice. Prospective studies with long-term followup are needed to determine whether the hybrid glenoid implant provides value to our patients.

Acknowledgements We thank the following people for help with the Hospital For Special Surgery shoulder arthroplasty registry: Sherrie Vassallo BA, Greg Mahony BA, Brian DePalma BA, Struan Coleman MD, Frank Cordasco MD, Joshua Dines MD, Stephen Fealy MD, Jo Hannafin MD, Anne Kelly MD, Bryan Kelly MD, John MacGillivray MD, Michael Maynard MD, Stephen O'Brien MD, Anil Ranawat MD, Scott Rodeo MD, Howard Rose MD, Beth Shubin Stein MD, Sabrina Strickland MD, Thomas Wickiewicz MD, Riley Williams MD; and Answorth Allen MD, David Altchek MD, Andrew Pearle MD, and Robert Marx MD (four of the surgeons cited in the Patients and Methods section of this article), all from the Hospital for Special Surgery (New York, NY, USA).

\section{References}

1. Baad-Hansen T, Kold S, Nielsen PT, Laursen MB, Christensen $\mathrm{PH}$, Soballe K. Comparison of trabecular metal cups and titanium fiber-mesh cups in primary hip arthroplasty: a randomized RSA and bone mineral densitometry study of 50 hips. Acta Orthop. 2011;82:155-160.

2. Barrett WP, Franklin JL, Jackins SE, Wyss CR, Matsen FA 3rd. Total shoulder arthroplasty. J Bone Joint Surg Am. 1987;69:865872.

3. Barwood S, Setter KJ, Blaine TA, Bigliani LU. The incidence of early radiolucencies about a pegged glenoid component using cement pressurization. J Shoulder Elbow Surg. 2008;17:703-708.

4. Bertollo N, Matsubara M, Shinoda T, Chen D, Kumar M, Walsh WR. Effect of surgical fit on integration of cancellous bone and implant cortical bone shear strength for a porous titanium. $J$ Arthroplasty. 2011;26:1000-1007.

5. Bohsali KI, Wirth MA, Rockwood CA Jr. Complications of total shoulder arthroplasty. J Bone Joint Surg Am. 2006;88:22792292.

6. Churchill RS, Boorman RS, Fehringer EV, Matsen FA 3rd. Glenoid cementing may generate sufficient heat to endanger the surrounding bone. Clin Orthop Relat Res. 2004;419:76-79.

7. De Wilde L, Dayerizadeh N, De Neve F, Basamania C, Van Tongel A. Fully uncemented glenoid component in total shoulder arthroplasty. J Shoulder Elbow Surg. 2013;22:e1-7.

8. Edwards TB, Labriola JE, Stanley RJ, O’Connor DP, Elkousy HA, Gartsman GM. Radiographic comparison of pegged and keeled glenoid components using modern cementing techniques: a prospective randomized study. J Shoulder Elbow Surg. 2010;19:251-257.

9. Flurin PH, Janout M, Roche CP, Wright TW, Zuckerman J. Revision of the loose glenoid component in anatomic total shoulder arthroplasty. Bull Hosp Jt Dis. 2013;71(suppl 2):68-76.

10. Fox TJ, Foruria AM, Klika BJ, Sperling JW, Schleck CD, Cofield RH. Radiographic survival in total shoulder arthroplasty. $J$ Shoulder Elbow Surg. 2013;22:1221-1227.

11. Franklin JL, Barrett WP, Jackins SE, Matsen FA 3rd. Glenoid loosening in total shoulder arthroplasty: association with rotator cuff deficiency. J Arthroplasty. 1988;3:39-46. 
12. Franta AK, Lenters TR, Mounce D, Neradilek B, Matsen FA 3rd. The complex characteristics of 282 unsatisfactory shoulder arthroplasties. J Shoulder Elbow Surg. 2007;16:555-562.

13. Fucentese SF, Costouros JG, Kühnel SP, Gerber C.Total shoulder arthroplasty with an uncemented soft-metal-backed glenoid component. J Shoulder Elbow Surg. 2010;19:624-631.

14. Gartsman GM, Elkousy HA, Warnock KM, Edwards TB, O'Connor DP. Radiographic comparison of pegged and keeled glenoid components. J Shoulder Elbow Surg. 2005;14:252-257.

15. Gregory T, Hansen U, Taillieu F, Baring T, Brassart N, Mutchler C, Amis A, Augereau B, Emery R. Glenoid loosening after total shoulder arthroplasty: an in vitro CT-scan study. J Orthop Res. 2009; 27:1589-1595.

16. Griffin JW, Hadeed MM, Novicoff WM, Browne JA, Brockmeier SF. Patient age is a factor in early outcomes after shoulder arthroplasty. J Shoulder Elbow Surg. 2014;23:1867-1871.

17. Hahn H, Palich W. Preliminary evaluation of porous metal surfaced titanium for orthopedic implants. J Biomed Mater Res. 1970;4:571-577.

18. Hasan SS, Leith JM, Campbell B, Kapil R, Smith KL, Matsen FA 3rd. Characteristics of unsatisfactory shoulder arthroplasties. $J$ Shoulder Elbow Surg. 2002;11:431-441.

19. Klepps S, Chiang AS, Miller S, Jiang CY, Hazrati Y, Flatow EL. Incidence of early radiolucent glenoid lines in patients having total shoulder replacements. Clin Orthop Relat Res. 2005;435: $118-125$.

20. Lazarus MD, Jensen KL, Southworth C, Matsen FA 3rd. The radiographic evaluation of keeled and pegged glenoid component insertion. J Bone Joint Surg Am. 2002;84:1174-1182.

21. Malizos KN, Bargiotas K, Papatheodorou L, Hantes M, Karachalios T. Survivorship of monoblock trabecular metal cups in primary THA: midterm results. Clin Orthop Relat Res. 2008;466:159-166.

22. Matsen FA 3rd, Bicknell RT, Lippitt SB. Shoulder arthroplasty: the socket perspective. J Shoulder Elbow Surg. 2007;16(5 suppl): S241-247.

23. Matsen FA 3rd, Clinton J, Lynch J, Bertelsen A, Richardson ML. Glenoid component failure in total shoulder arthroplasty. $J$ Bone Joint Surg Am. 2008;90:885-896.
24. Olson S, Clinton JM, Working Z, Lynch JR, Warme WJ, Womack W. Matsen FA 3rd. Thermal effects of glenoid reaming during shoulder arthroplasty in vivo. J Bone Joint Surg Am. 2011;93:11-19.

25. Papadonikolakis A, Neradilek MB, Matsen FA 3rd. Failure of the glenoid component in anatomic total shoulder arthroplasty: a systematic review of the English-language literature between 2006 and 2012. J Bone Joint Surg Am. 2013;95:2205-2212.

26. Rasmussen JV, Polk A, Brorson S, Sorensen AK, Olsen BS. Patient-reported outcome and risk of revision after shoulder replacement for osteoarthritis: 1,209 cases from the Danish Shoulder Arthroplasty Registry, 2006-2010. Acta Orthop. 2014;85:117-122.

27. Tammachote N, Sperling JW, Vathana T, Cofield RH, Harmsen WS, Schleck CD. Long-term results of cemented metal-backed glenoid components for osteoarthritis of the shoulder. $J$ Bone Joint Surg Am. 2009;91:160-166.

28. Taunton MJ, McIntosh AL, Sperling JW, Cofield RH. Total shoulder arthroplasty with a metal-backed, bone-ingrowth glenoid component: medium to long-term results. J Bone Joint Surg Am. 2008;90:2180-2188.

29. Throckmorton TW, Zarkadas PC, Sperling JW, Cofield RH. Pegged versus keeled glenoid components in total shoulder arthroplasty. J Shoulder Elbow Surg. 2010;19:726-733.

30. Vavken P, Sadoghi P, von Keudell A, Rosso C, Valderrabano V, Muller AM. Rates of radiolucency and loosening after total shoulder arthroplasty with pegged or keeled glenoid components. J Bone Joint Surg Am. 2013;95:215-221.

31. Vidil A, Valenti P, Guichoux F, Barthas JH. CT scan evaluation of glenoid component fixation: a prospective study of 27 minimally cemented shoulder arthroplasties. Eur J Orthop Surg Traumatol. 2013;23:521-525.

32. Wirth MA, Loredo R, Garcia G, Rockwood CA Jr, Southworth C, Iannotti JP. Total shoulder arthroplasty with an all-polyethylene pegged bone-ingrowth glenoid component: a clinical and radiographic outcome study. J Bone Joint Surg Am. 2012;94:260-267.

33. Wirth MA, Rockwood CA Jr. Complications of total shoulderreplacement arthroplasty. J Bone Joint Surg Am. 1996;78:603616. 